\title{
Die Dauer der postmortalen mechanischen Muskelerregbarkeit bei chronischen Geisteskranken, speziell Paralytikern.
}

\author{
Von \\ Medizinalrat Prof. Dr. P. Näcke (Hubertusburg). \\ (Eingegangen am 12. September 1911.)
}

Wir wissen, daß mit dem Eintritt des Todes die Organe und ihre Gewebe nicht sofort absterben, sondern ihre Funktion erst nach einer gewissen Zeit einbüßen. Das bezieht sich insbesondere auch auf die Körpermuskulatur. Leider gibt es aber hierüber nur sehr weniges in der Literatur. Ich finde eigentlich bloß in einer Arbeit von Toulouse und Vurpas ${ }^{1}$ ) einen Hinweis, daß man normalerweise nach dem Tode (in einem Falle sogar 3 Stunden p. m.) noch fibrilläre Zuckungen oder Muskelknoten (nouds) antrifft, aber keine Zuckungen mehr oder weniger des ganzen Muskels, wie in vivo. Mangold ${ }^{2}$ ) hat die Erregbarkeit des herausgeschnittenen Muskels in physiologischer oder 6 proz. CINa-Lösung untersucht. Dies würde uns hier aber nicht weiter interessieren.

Untersuchungen über die postmortale mechanische Muskelerregbarkeit bei Geisteskranken sind aber überhaupt nicht bekannt, soviel ich sehe, und so glaube ich mich hier auf vollkommenem Neuland zu bewegen. Die Frage selbst erscheint nicht nur biologisch interessant, sondern sie hat wahrscheinlich auch einen nicht zu unterschätzenden forensischen Wert zur Bestimmung, wie lange höchstens der Tod eines bestimmten Individuums erfolgt sein könnte. Dies soll nun im folgenden an einem relativ großen Material untersucht werden, wobei freilich nur zu bedauern ist, daß Vergleichswerte an Normalen uns gänzlich fehlen, so daß die gefundenen Zahlen zurzeit noch in der Luft schweben und dann erst wirklich wertvoll sich erweisen werden, wenn die gleichen Experimente bei gleicher Methodik auch an Normalen gemacht und registriert worden sind. Die vorliegenden Ergebnisse

1) Toulouse et Vurpas: Etude synthétique des réactions musculaires dans la paralysie générale usw. Revue de Psych. 1903, S. 502.

2) Mangold: Über die postmortale Erregbarkeit quergestreifter Warmblütermuskeln. Arch. f. d. ges. Physiol. XXVI., S. 498, nach einem Ref. im Arch. di Psich. etc. 1904, S. 766. 
sind also nur künftig erst voll zu verwerten, lassen aber immerhin schon jetzt interessante Einblicke in gewisse Verhältnisse zu.

Untersucht wurden 30 chronisch geisteskranke Männer, ohne Auswahl, außer daß solche, deren Todesstunde zu einer mehrfachen Untersuchung unbequem lag, andere wieder aus besonderen Gründen, ausgelassen wurden. Die meisten stammten aus den Jahren $1904-06$. Alles Nähere ist in den angehängten Tabellen angegeben. Hier wollen wir nur Zusammenfassungen geben. Im Alter von 30-40 Jahren standen 8 Männer, im Alter von 40-50 Jahren 11, darüber 11. Der Jüngste war $33^{3} / 4$ Jahre, der Älteste 74 Jahre. Bis auf 2 Ärzte und 3 Kaufleute gehörten alle den niederen Volksschichten an, mit fast nur Elementarbildung. Die Dauer der Anstaltsbehandlung (allein in der hiesigen Anstalt oder zusammen mit der in einer anderen) betrug 4 mal bis zu 3 Monaten, 5 mal 3 Monate bis zu 1 Jahre, 4 mal 1-2 Jahre, 5 mal 2-3 Jahre, 5 mal 3-5 Jahre und 7 mal darüber. Bezüglich der Diagnose gab es 18 Paralytiker (davon 2 allerdings nicht ganz sicher, weil damals $W$. noch nicht gemacht wurde), 4 an Dementia praecox Leidende, je 2 an Bleipsychosen und Paranoia Erkrankte [1 mal chronische Paranoia, das andere Mal chronische halluzinatorische Verrücktheit, die eventuell der Dementia-praecox-Gruppe beigezählt werden müßte $\left.{ }^{1}\right)$ ] und je 1 an Idiotie, Dementia senilis, Melancholia senilis und Intoxikationspsychose (Urämie).

Da anzunehmen war, daß der Ernährungszustand nicht ganz ohne Einfluß wäre, ward auch hierauf geachtet und $18 \mathrm{mal}$ die Leiche stark abgemagert, 8 resp. 9 mal nicht und 4 resp. 3 mal mehr oder weniger ödematös gefunden. Untersucht war der Tote $1 \mathrm{mal}$ in $1 \mathrm{Falle}, 2 \mathrm{mal}$ in 11, 3 mal in 16 und $4 \mathrm{mal}$ in 2 Fällen, in der Mehrzahl also 3 und 2 mal. Die erste Explorierung selbst erfolgte 2 mal 10 Minuten p. m., $11 \mathrm{mal}$ 10-30 Minuten, $8 \mathrm{mal} 30$ Minuten, $5 \mathrm{mal} 1 / 2-1$ Stunde p. $\mathrm{m}$. und $4 \mathrm{mal}$ darüber $\left.^{2}\right)$. Sie geschah also meist 10-30 Minuten, dann 30 Minuten p. m.; frühestens 10 Minuten, spätestens (aus äußeren Gründen) 2 Stunden (in Nr. 9). Die Zeit der letzten Untersuchung war 19 mal $1-2$ Stunden p. m., 9 mal $2-3$ Stunden und 1 mal darüber, in 29 Fällen (da hier Nr. 9 nicht mitgezählt ist).

Wir wenden uns jetzt der Methodik zu. Tagsüber sind die Untersuchungen leichter anzustellen als nachts. Man zieht das Bett mit der Leiche möglichst nahe an das Fenster, so daß das volle Tageslicht auf die perkutierte Muskelpartie fällt. Dies gilt besonders vom Gesichte, da es sich oft genug um kaum wahrnehmbare Zuckungen usw. handelt. Nachts bringe man eine elektrische Birne ganz nahe an den zu prüfen-

1) Chr. Saiz: Dem. praecox u. Paranoia hallucin. chron. Allgem. Zeitschr. f. Psych., Bd. 68 (1911), S. 447 usw.

2) Darunter auch Nr. 9, wo nur überhaupt ein einziges Mal untersucht wurde. 
den Muskel heran. Schon deshalb muß ein Wärter behilflich sein, noch mehr aber, um die Leiche umzuwenden, wenn man die Seiten und die Rückenpartie explorieren will. Man benutzt einen gewöhnlichen Perkussionshammer, am liebsten einen schweren, ohne Plessimeter. Das Kneifen des Muskels habe ich nicht vorgenommen. Man schlägt nun mit dem Hammer senkrecht auf den Muskel, schnell und gewöhnlich kurz. Auch der Knochen wird direkt angeschlagen. Es ist nun gewiß nicht leicht, immer die gleiche Stärke des Aufschlags beizubehalten, und danach ändert sich oft genug der Befund $\left.{ }^{1}\right)$. Die letzten Reaktionen des absterbenden Muskels kann man aber nur durch sehr kräftiges, elastisches und langes Anschlagen erzielen, während vorher geringere Stärkegrade meist schon genügen. Vielleicht ist es jedoch besser, was ich freilich nicht immer tat, gleich von vornherein kräftig zu schlagen, da man dann noch gewisse Reaktionen erzielt, die sonst nicht erscheinen. Am besten wäre aber die Anwendung eines Apparates, der stets sicher die gleiche Schlagstärke verbürgt, sie zahlenmäßig angibt, sie auch zahlenmäßig steigern läßt und stets in gleicher Richtung, in gleichem Winkel aufschlägt. Dadurch ließen sich verschiedene Fehlerquellen schon beseitigen. Nicht unwesentlich erscheint es auch, stets de nselben Hammer zu benutzen, da sonst leicht Differenzen entstehen. Für spurweise Reaktionen hatte ich anfangs eine Lupe angewandt, bin aber davon bald abgekommen, da sie die Untersuchung erschwerte und kaum mehr angab als das bloße Auge. Das Resultat wird dann auch dadurch getrübt, daß man nicht gut senkrecht anschlagen kann. Da die Beurteilung des Eintritts einer minimalen Reaktion, ebenso die Abschätzung der Größe derselben mehr oder minder subjektiv bleibt, ist es hier, wie bei ähnlichen Untersuchungen, am besten, wenn zwei Personen gleichzeitig arbeiten und sich so gegenseitig kontrollieren. Wer, wie ich, sehr kurzsichtig ist, hat noch einen besonders schlimmen Stand.

Eine weitere Schwierigkeit in der Beurteilung liegt im Ernährungszustande der Leiche. Je abgemagerter dieselbe ist, um so stärker müssen a priori im allgemeinen die Reaktionen sein, und insofern spielt gewiß auch die Todesursache mit, vielleicht auch die Form und Dauer der Psychose ${ }^{2}$ ). Bei nicht Abgemagerten, gar noch bei Hydropischen, muß man, um gleiche Erfolge zu erzielen, um so stärker zuschlagen,

1) So passierte es mir z. B., daß ich bei der 1. Untersuchung an gewissen Muskeln keine Zuckungen usw. sah, wohl aber bei der nächsten, wenn auch nur schwach; oder der 2. Ausschlag war stärker als der erste. Offenbar in beiden Fällen hauptsächlich, weil nicht gleichstark angeschlagen wurde.

2) Ist die Abmagerung durch Ernährungsstörung infolge von Krankheiten aller Art entstanden, so wird nach Toulouse (l. c.) die muskuläre Reaktion schon in vivo keine normale sein, sondern fribrilläre und knotenartige Zuckungen ergeben. 
und es ist dann natürlich schwer, richtige Vergleiche anzustellen. Bei Fetten wird an sich oft genug der Muskel schwächer als normal sein, also schwächer reagieren, und die überliegende Fettschicht hemmt natürlich die Einwirkung des Schlages. Bei Hydrops wiederum bildet die seröse Durchtränkung des subcutanen Bindegewebes und der Muskeln gleichfalls Hindernisse, die in concreto schwer abschätzbar sind. Dasselbe geschieht bei Eintritt der Totenstarre, deren Grade sehr verschieden sind und demnach die Funktion der Muskeln verschieden affizieren. Der Untersucher schlägt aber auch nicht immer unter gleichem Winkel und gleich stark. Es ist weiter nicht gleich, wo man hinschlägt, ob oben, unten, in der Mitte oder auf der Seite des Muskels, auf die Sehne oder auf den Knoehen, gerade von oben, oder mehr seitlich.

Man sieht also, der z. T. kaum behebbaren Fehlerquellen gibt es viele, wenn man ganz minuziös verfahren will, was ich daher für fast unmöglich halte. Und weitere Fehlerquellen können wir höchstens nur ahnen. So ist es nicht unwahrscheinlich, daß Rasse, Temperament, Alter usw. eine gewisse Rolle spielen. Für unsere $Z$ wecke genügen aber wohl schon gröbere Unterschiede, die mit bloßem Auge und gröberer Technik sichtbar sind, ebenso die Angabe größerer Zeitabschnitte, nicht also bis auf Minuten und Sekunden. Die positive Reaktion habe ich in der Tabelle mit +, die negative mit - und die Reaktionsstärke der positiven Fälle mit 2,3 bezeichnet, was freilich immer etwas willkürlich bleibt. Im allgemeinen ist bei jeder späteren Untersuchung das Zeichen + als schwächer anzusehen als bei der vorhergehenden, und bei der letzten Prüfung bedeutet es meist nur eine spurweise, eben noch sichtbare Reaktion.

Wenn wir jetzt die Reaktionen selbst kurz besprechen wollen, so darf man zunächst nicht bloße Erschütterungen der Haut mit Zuckungen verwechseln, wie es $\mathrm{z}$. B. bei hydropischen oder sehr fetten Leichen bei starkem Anschlagen wohl einmal geschehen kanm. Die Kontraktionen betreffen sehr selten den ganzen Muskel oder einen großen Teil desselben zu gleicher Zeit ${ }^{1}$ ). Es kontrahieren sich vielmehr immer nur wenige Bündel gleichzeitig, wie man dies am besten am M. deltoid.

1) Doch sah ich wiederholt starke Kontraktionen zwischen den Schulterblättern, wenn man den Intersca pularraum beklopfte, oder das eine Schulterblatt hob sich deutlich in die Höhe. Sehr selten wurden durch Beklopfen des Vorderarmes innen die Hand oder gar die Finger bewegt, dagegen sehr oft der Daumen, wenn man den Thenar perkutierte, seltner war dies am Anthenar, bez. der letzten Finger zu sehen. Einmal hob sich beim Anschlagen des Oberschenkels die Kniescheibe deutlich, öfter ward oin Emporziehen der Augenbrauen konstatiert. Ein merkwürdiges Verhalten zeigte ein Taubstummer (nicht in unserer Tabelle mit aufgenommen). Schon nach 15-20 Minuten waren Totenflecke da uud beim Aufheben des rechten Vorderames hüpften deutlich spontan (also ohne Anklopfen) die Muskeln an der Innenfläche des Vorderarmes. 
und pector. sehen kann. Die Zuckungen selbst sind verschieden lang, hoch, treten sogleich oder erst nach einiger Zeit auf, vergehen schnell oder können längere Zeit bestehen bleiben, sind öfters rechts und links verschieden stark ausgeprägt ${ }^{1}$ ), sie können auf einer Seite sogar einmal fehlen. Mit der Länge der Zeit und fortschreitender Starre werden sie immer oberflächlicher, seichter und verkürzen sich. Die dünnen Muskeln an der Stirn, im Gesicht, zwischen den Rippen oder Phalangen verlieren im allgemeinen schneller ihre Erregbarkeit als die dickeren und von letzteren wieder die unteren Extremitäten eher als die oberen. Am Bauche sah ich nur ein einziges Mal ganz oberflächliche Zuckungen, etwas häufiger am Rücken unter der Scapula. Die Zuckung bildet einen ganz schmalen, länglichen Wulst unter dem Hammer, sehr selten eine Rinne, indem scheinbar nicht der getroffene Teil sich erhebt, sondern die angrenzenden Partien rechts und links ${ }^{2}$ ). Die letzten Spuren der Zuckungen sind schwer zu sehen und namentlich von Erschütterung der Haut nicht leicht zu trennen.

Mit der Zeit verkürzen sich, wie gesagt, immer mehr die Z. (= Zukkungen), und es tritt statt derselben, nicht selten aber auch gleichzeitig, im selben Muskel oder im angrenzenden, ein mehr oder weniger elliptischer Muskelwulst, Muskelknoten ${ }^{3}$ ) auf (Toulouse nennt ihn Noud, Knoten), und zwar in vertikaler oder querer Richtung, und häufiger an den starken Muskeln auftretend. Er ist verschieden nach Länge (ich sah solche bis zu 3-4 cm Größe), Höhe, Richtung, Schnelligkeit im Auftreten und Vergehen, je nach der Zeit post mortem, der fortschreitenden Starre und je nach der Stärke des Schlags. Er wird, im Gegensatz zu den Z., mit der Zeit nicht kürzer, sondern länger, aber auch flacher, und er kann bis zu 1/2 Stunde, 1 Stunde, vielleicht auch noch länger nach dem Schlage stehenbleiben, nach Art eines Tetanus des Muskels. Die Wulstbildung geschieht meist in der Längsrichtung des Muskels, also z. B. vertikal beim M. deltoid. und pector., doch sah ich hier auch quere entstehen, sogar bisweilen mit jenen zusammen. Die queren erkläre ich mir dann durch Zusammenfließen von nebeneinander getroffenen, erregbaren, sehr kurzen Bündelteilen oder aber durch

1) Links sind sie sehr oft schwächer und eher verchwindend als rechts.

2) Vielleicht kontrahiert sich aber doch das getroffene Bündel, nur später als die andern, so daß es sich erhebt, wenn die seitlichen Partien vergehen. Beobachtet habe ich es aber selbst nicht.

3) In den Tabellen ist er mit o bezeichnet und seine Stärkegrade mit 2,3. Es fragt sich, ob er in allen Muskeln vorkommt, was mir nicht wahrscheinlich ist. Mann könnte dann vielleicht annehmen, daß er durch Absterben des Muskels zu gleicher Zeit proximal und distal und durch Erhalten der erregbaren Mitte ent. steht, bei den andern Muskeln dagegen das Absterben nicht von oben und unten zugleich, sondern von oben nach unten zu geschieht, die Zuckung immer kleiner wird, es also in der Mitte nicht mehr zur Bildung eines Wulstes kommen kann, höchstens vielleicht am Ende des Muskelbündels. 
starke Kontraktion eines oder nur sehr weniger Bündel, wodurch die quere Richtung entsteht.

Der Wulst ist also jedenfalls ein s päteres Stadium des Absterbens, wenn nur noch in der Mitte kontraktionsfähige Masse übrig bleibt. Der quere Wulst oder besser gesagt, der zur Faserrichtung senkrechte, scheint mir im allgemeinen später aufzutreten als der entgegengesetzte. Bisweilen fanden sich $\mathrm{Z}$. und Wülste zugleich am selben Muskel oder an verschiedenen. Je kräftiger der Körper noch ist, desto mehr Z. treten auch weitverbreitet auf, ebenso je stärker geschlagen wird. Mit dem Absterben nimmt umgekehrt die Zahl und Höhe der Wülste zu, ebenso mit der Schlagstärke.

Verkürzt sich der W. (= Wulst) noch mehr, so bleibt nur ein kleiner, runder, also nicht mehr ovaler Hügel übrig, eine förmliche Quaddel. Diese Bildung ist sehr selten ${ }^{1}$ ). Auch sie kann Verschiedenheiten darbieten und lange bestehen bleiben. Als letztes Lebenszeichen tritt endlich die Dellenbild ung auf, wobei unter dem Hammer eine Delle sich zeigt - am häufigsten am M. pector., sehr selten deltoid., öfter am Front. und am Sternum - und zwar dadurch, daß ringsherum ein muskulöser Wall ensteht. Auch hier gibt es Varianten. Sie kann, wie der Wulst, längere Zeit bestehen bleiben, auch asymmetrisch oder einseitig sein. Die letzten Spuren sind auch hier schwer zu erkennen. Deutlich von dieser Dellenbildung ist aber eine andere zu trennen - mitunter freilich schwierig genug - nämlich die durch den Verlust der Hautelastizität entstandene, abgesehen natürlich von ähnlichen Bildungen bei Ödemen²).

Was die Entstehung der postmortalen Z. und W. anbelangt, so gilt wohl auch der Satz, wie beim Muskel in vivo, daß nämlich dabei nicht nur die Muskelsubstanz selbst beteiligt ist, sondern auch der Nerv, der freilich sehr wahrscheinlich seine Vitalität viel schneller einbüßt, als der Muskel. Diese in beiden Substanzen abnehmende Reaktionsfähigkeit zeigt sich in dem Auftreten von vorwiegend fibrillären Z., die anfangs schnell eintretend und lang sind, bald aber sich immer mehr verflachen und verkürzen, dafür aber länger beharren, bis zuletzt nur der W. und die Delle übrig bleiben. W. und Delle ihrerseits weisen ähnliche Veränderungen infolge abnehmender Vitalität auf. Gerade der W. neigt sehr zum tetanusartigen Krampf, viel mehr als die $\mathbf{Z}$. Warum er viel häufiger asymmetrisch oder einseitig ist als jene, ist

1) Ich frage mich, ob nicht vielleicht eine beklopfte Stelle in ähnlicher Weise auch erst später sich erheben kann, wenn der Untersucher schon fort ist, oder ob etwa solche runde W. sogar in angrenzenden Teilen sich bilden können.

2) Einmal sah ich beim Aufschlagen auf die Tibia und außen davon überall, wo der Hammer aufgetroffen war, runde, von der Epidermis entblößte Stellen, aber ohne Muskelwall. 
schwer zu sagen ${ }^{1}$ ). Eher schon, warum bisweilen $Z$. u. W. gleichzeitig im selben oder benachbarten Muskeln sich zeigen, weil offenbar die einzelnen Muskeln und hier wieder die einzelnen Bündel zu verschiedenen Zeiten absterben.

Die beschriebenen Erscheinungen am Muskel sind aber auch schon in vivo zu sehen, wie dies besonders Toulouse (1. c.) und Bechterew ${ }^{2}$ ) betonen, und zwar bei allerlei schweren Ernährungsstörungen infolge akuter und chronischer Leiden, speziell Nerven- und Geisteskrankheiten. Auch experimentell wurde die Sache erhärtet. Toulo use unterscheidet zwei „vies musculaires", die gewöhnlich übereinandergelagert sind: 1. une vie propre et autonome, die auch einige Zeit nach dem Tode noch anhält und sich in langsamen, flachen, bündelweisen $\mathrm{Z}$. dokumentiert (nach dem Typus des glatten Muskels; Näcke) und 2. une vie synthétique et solidaire du système nerveux, wobei die Muskeln mehr in toto schnell und stark sich zusammenziehen (nach dem Typus des gestreiften Muskels; Näcke). Bei gewissen Nerven- und Geisteskranken - insbesondere bei der Paralyse - tritt nun schon in vivo die Reaktion nach dem Typus des glatten Muskels auf, wie sonst nach dem Tode, und es findet somit eine Regression auf einen früheren Entwicklungszustand statt, da der Typus des glatten Muskels phylogenetisch der ältere ist. Marie ${ }^{3}$ pflichtet diesen Ausführungen Toulo uses bei. Andererseits sehen wir bei gewissen Nerven- und Gemütsleiden Steigerung der Muskelerregbarkeit in vivo eintreten, z. B. der Sehnenreflexe, des Facialisreflexes bei Paralyse. Letzteren überschätzt aber offenbar To ulo use sehr. Ich habe speziell auf diesen Punkt geachtet und habe durch starkes Beklopfen des Kinns keine häufigere Kontraktion der Kinn- und Mundmuskeln gesehen, als z. B. bei Dementia praecox. Auch Bechterew spricht sich ähnlich aus. Dagegen fand ich hier - allerdings auch bei anderen Psychosen in vivo-sehr häufig fibrilläre Muskelzuckungen am Pector., Deltoid, den Armmuskeln, seltener an den Bein-, am seltensten an den Bauchmuskeln und die Erregbarkeit erhöht. Doch das nur nebenbei. Als Muskelreflex bezeichnet Bechterew nur die Kontraktion des ganzen Muskels oder eines Bündels, als idiomuskuläre Kontraktibilität oder Muskelwulst, den Klippel und Toulouse auch réflexe idiomusculaire ou myo-oedème nennen, dagegen die $\mathbf{Z u}$ sammenziehung des Muskels unmittelbar an der beklopften Stelle selbst. Bei manchen Krankheiten zeige sich beides. Ich selbst sah es so einige Male

1) Vielleicht zeigt sich hierin die ungleichmäßige Entwicklung der Muskeln rechts und links nach mehr als bei der $\mathrm{Z}$.

2) Bechtere w: Úber die Untersuchung der mechanischen Muskelerregbarkeit und der sogenannten Muskelreflexe und ihre Bedeutung. Neurol. Centralbl. 1906, S. 1090.

3) A. Marie. La démence. Paris 1906, S. $166 \mathrm{ft}$. 
in vivo bei Paralytikern am M. pectoralis, ob auch bei anderen Irren, weiß ich nicht mehr. Der Muskelreflex ist nach Bechterew etwas anderes als der Sehnenmuskelreflex, da letzterer fehlen kann, wenn ersterer noch da ist. Der erstere ist auch am herausgeschnittenen Muskel zu beobachten. Nach Bechterew ist der Muskelreflex bei verschiedenen Krankheiten der Nerven erhöht. Als neu fand er diesen bei Affektionen des Plexus cervicalis oder vielen Armnerven gesteigert am Biceps, Triceps, wobei zugleich die Zuckungen ,auffallend welk und langsam" sich vollzogen - wie ich dies offter bei Paralyse sah -, ebenso im atrophischen Muskel, ein Analogon zur EA, die dann nie fehlt. Die größte mechanische Muskelerregbarkeit sieht man bekanntlich bei Tetanie. Hier konnte Bechterew bisweilen bei einigen Muskeln (Gesicht, Arm) allmähliches Anwachsen der Stärke der Reflexe bei wiederholtem Beklopfen derselben Stelle ermitteln, was ich bei lebenden Geisteskranken, speziell Paralytikern, nie sah, auch nicht nach dem Tode.

Die in unseren Fällen untersuchten Muskeln waren nun folgende. An der Stirn wurde der M. front. und corrugator supercilii beklopft. Einige Male zog sich der letztere zusammen bei Perkutieren des Arcus superciliaris. An der Stirn zeigten sich viel seltener niedere W. oder Dellen, als Z. Am Gesicht ward der Arcus infraorbit., die Jochbeine, die seitlichen Partien der Nase und besonders das Kinn beklopft. Der Orbicularis zeigte geringe Neigung, sich zusammenzuziehen, noch viel seltener aber die kleinen Gesichtsmuskeln, so daß der Mundwinkel sich hob. Auch am Kinn waren Z., im Gegensatz zum Leben, sehr selten, doch mag der Bart dieselben öfters verdeckt haben, weshalb es sich empfiehlt, hier besonders stark zu schlagen. Am Halse ward der Kinnbacken, die Gegend seitlich am Kehlkopfe und unterhalb des Ohres angeschlagen. Der M. cucullaris (platysma?) reagierte nur einmal deutlich, öfter dagegen der sternocleido-mastoideus. An der Brust waren die Schlagstellen das Schlüsselbein, das Brustbein, der M. pector. und die Rippen seitlich (M. serratus ant. maj. und Latissimus dorsi in der Hauptsache). Beim M. pector. zogen sich immer nur einzelne Bündel zusammen, am meisten und zuletzt außen und in der Mitte, oder es bildete sich (auch sonst bisweilen) sehr selten eine Rinne, wie sie Bechterew bei gewissen Fällen von Myotonie sah. Nie ward der Arm in die Höhe gezogen, wohl aber öfter die Achselfalte. Am Sternum zeigten sich oft Dellen, seltener Wülste. Sehr selten waren Z . in den Zwischenrippenräumen, fast nie am Bauche. Am Arme wurden besonders der M. deltoid. und biceps perkutiert. Auch hier wurden nur $Z$. und $W$. erzeugt, nie Armbewegungen. Beim M. deltoid. waren die $Z$. am meisten, am frühesten und bis zuletzt in den oberen äußeren Teilen sichtbar. Die beklopfte Innenfläche des Vorder- 
arms zeigte nur selten Z., aber einige Male mit Hand- und sogar Fingerbewegungen. Auch am Radius ward angeschlagen. Der perkutierte Daumenballen (Thenar) brachte fast stets oder sehr häufig wenigstens $Z$. hervor, auch W., sogar mit Daumenbewegungen, viel seltener war dies am Antithenar zu sehen. Die Interossei zeigten selten Z., öfter die Muskeln der Außenseite des Vorderarms. An den Beinen ward der Quadriceps und die Außen- und Innenseite untersucht. Im allgemeinen zeigten sich Z. öfter an der Innen- als Außen- und Vorderseite. Selten waren Z. außen von der Tibia. Auf der Rückseite war der Nacken (bes. des Cucullaris und Splenius) häufiger der Sitz von Zuckungen als die Partien seitlich am Halse, immerhin selten genug. Viel öfter dagegen waren solche zwischen den Schulterblättern (Cucull. und Serratus ant. maj.), wodurch sie einander genähert wurden, wenn man den Interscapularraum beklopfte, oder der obere Winkel derselben zog sich gar in die Höhe (M. levator scap., rhomboid.). Seltener zogen sich die M. supra- und infraspinati bei Perkussion der Gruben der Scapula zusammen und bildeten tiefe Dellen. Seitlich an den Rippen zeigten sich fast nie Z., häufiger dagegen in den M. glutaei., besonders auf der Höhe (M. glut. maxim.). Man mußte hier aber stets stärker zuschlagen. An der Rückseite der Oberschenkel (Hauptmuskel der Biceps fem.) waren nur selten $Z$. zu sehen, ebenso an dem Nacken. Nur einmal hoben sich beim Beklopfen des Fußrückens die Zehen.

Es kann nun nicht unsere Absicht sein, hier alle die vielen Möglichkeiten und interessanten Details des näheren anzuführen. Das würdo viel zu weit führen, und außerdem findet sich das Nötigste darüber in den Bemerkungen der angehängten Tabellen. Wir wollen dagegen einige Utberblicke geben, die uns gewisse Richtungslinien erkennen lassen. Hierbei werden wir aber nicht die einzelnen Muskeln berücksichtigen, sondern, etwas summarisch allerdings, bestimmte Körperregionen.

Zunächst sehen wir, daß in nur 6 Fällen von $29^{1}$ ) (Nr. 1, 2, 14, 16, 19, 22) bei der letzten Perkussion nichts mehr zu sehen war, weder $\mathrm{Z}$. noch W.; am frühesten nach 1 Stunde 20 Minuten, am spätesten meist zwischen 2-3 Stunden. Bei all den übrigen Fällen war immer nach der letzten Untersuchung noch einiges Muskelleben nachweisbar, und zwar hörten im allgemeinen die Z. eher auf als die Wülste. Die letzten beobachteten $\mathrm{Z}$. waren 9 mal zwischen $1 / 2-1$ Stunde p. m., 10 mal zwischen $1-1 \frac{1}{2}$ Stunden. 5 mal zwischen $1 \frac{1}{2}-2$ Stunden, 2 mal zwischen $21 / 2-3$ Stunden. am spätesten nach $23 / 4$ Stunden. Es läßt sich also hier zwar nicht genau der Zeitpunkt bestimmen, wann die Z. wirklich aufhörten, da weitere Untersuchungen nicht gemacht wurden,

1) Nr. 9 nicht eingerechnet, weil er nur einmal und zwar 2 Stunden p. m. untersucht ward und nur $W$. dann sich vorfanden. 
aber viel länger als 3 Stunden werden sie kaum angedauert haben. Man kann also die Grenze wohl zwischen 3 und 4 Stunden setzen. Muskelwülste wurden, wie gesagt, meist noch gesehen, wenn die Z . verschwunden oder im Verschwinden begriffen waren. Sie entstanden meist auch später. Die letzten W. wurden gesehen in der Zeit bis zu 11/2 Stunden in 18 Fällen, bis zu 2 Stunden in 3 , bis zu $2 \frac{1}{2}$ Stunden in 4 und darüber hinaus in weiteren 4 Fällen. Schon ein flüchtiger Blick in die Tabellen zeigt ferner, wie mit den fortschreitenden Untersuchungen und eintretender Totenstarre die Z. immer seltener, die W. dagegen an Zahl und Ausbreitung häufiger werden; auch in einem und demselben Muskel geschah dies. In 6 Fällen unter 26 waren sie zuletzt allein da, während Z. allein in nur 3 Fällen sich zeigten, was möglicherweise mit Fehlern der Methodik zusammenhing. In allen übrigen Fällen standen zuletzt noch Z. u. W. nebeneinander, wobei jedoch wiederum letztere an Zahl weit überwogen. Wären hier noch weitere Prüfungen vorgenommen worden, so hätten sicherlich nur W. den Platz behauptet. In einem Falle (Nr. 9) waren überhaupt nur Wülste da, was sich aber einfach daraus erklärt, daß nur eine einzige Untersuchung, und zwar 2 Stunden p. m. stattfand, zu einer Zeit also, wo sehr wahrscheinlich die Z. schon vergangen waren, zumal es sich um eine ödematöse Leiche handelte. In einem anderen Falle (Nr. 22) zeigten sich nur Z., vielleicht waren aber hier zugleich oder später leichte $W$. da, aber übersehen worden.

Úber die Häufigkeit der Z. u. W. in den einzelnen Regionen sollen folgende Zahlenangaben Auskunft geben. Freilich geben sie, wie auch sonst, nur die ungefähren Verhältnisse wieder, da 1. leicht ganz schwache $Z$. und W. einmal übersehen werden können, 2. nicht immer gleich stark angeschlagen ward, wodurch gewisse Differenzen entstanden, und 3. die Beurteilung der Stärkegrade der Erzeugnisse mehr oder minder subjektiv bleibt, wohl sogar bei einem und demselben Untersucher wechseln kann. Wir fanden so unter 29 Fällen an der Stirngegend Z. 16 mal (darunter 1 mal stark), am Gesicht 19 mal (3 mal stark), an der Brust $22 \mathrm{mal}$ (5 mal stark und 1 mal sehr stark), an den Armen $26 \mathrm{mal}$ (3 mal stark), an den Beinen 24 mal (5 mal stark), am Nacken 11 mal (1 mal stark), am Rücken 21 mal (5 mal stark) und am Gesäß $15 \mathrm{mal}$ (6 mal stark). Am Nacken, Rücken und Gesäß würde sich die Zahl wohl noch erhöhen, da diese Gegenden in 4 Fällen nicht untersucht wurden. Immerhin zeigt sich klar, daß die Arme am häufigsten, dann die Beine, also die Extremitäten von $\mathrm{Z}$. betroffen wurden, gleich darauf die Brust, der Rücken und das Gesicht kamen, während das Gesäß wenig Z. aufwies, am wenigsten aber Stirn und Nacken. Dem entspricht auch ungefähr die Zahl der starken Z.: an den Armen 50\%, an den Beinen $20 \%$, an Brust $22 \%$, am Rücken $24 \%$, Gesäß $40 \%$, Stirn $61 / 4 \%$, Nacken $9 \%$, nur daß hier das Gesäß nach den Armen die meisten stärksten 
Grade zeigte. Im allgemeinen folgte auch die Häufigkeit der Z. in ein und demselben Muskel dieser Regel, ebenso schien die Stärke des Ausschlags damit parallel zu gehen. Bez. der Ausbreitung auf verschiedene Körperteile wurden 2 mal 2 Regionen, 2 mal 3 (davon 1 mal stark), 4 mal 4, 2 mal 5, 6 mal 4 (darunter 3 stark), 4 mal 7 ( 3 stark), 7 mal 8 (6 mal stark) unter 25 Fällen von $Z$. betroffen. Wir sehen also, daß die Ausbreitung über 6-8 Regionen vorwiegt, die über 8 aber alle überragt und mit dem geht dann auch die Stärke der Z. im allgemeinen Hand in Hand. Auch dort, wo weniger Körperteile (2-4) betroffen wurden, überwogen gleichfalls an Häufigkeit der Z . wieder die Extremitäten (besonders die oberen) und die Brust.

Betrachten wir nun die Muskelwülste, so liegen die Verhältnisse ähnlich. In 30 Fällen (bei 4 allerdings nicht die Rückfläche untersucht) waren sie am häufigsten am Arme (23 mal, darunter 6 mal stark), dann an der Brust (20 mal, 5 mal stark), an den Beinen 11 mal (2 mal stark), am seltensten am Rücken (9 mal, 1 mal stark), an der Stirn (7 mal, 1 mal stark), am Gesäß (4 mal, 2 mal stark) und am Nacken (1 mal, und dies stark). Also auch hier stehen die Extremitäten und die Brust an der Spitze. Der Stärke nach waren sie am häufigsten am Gesäß $(50 \%)$ und dann ca. $25 \%$ bei Gesicht, Brust und Arm, am wenigsten am Nacken. Es ist also auch hier (außer am Gesäß) ein gewisser Parallelismus vorhanden. Einseitig fanden sich W. nur 1 mal an (Nr. 2). Der Zahl der betroffenen Regionen nach fanden sie sich in 25 Fällen am häufigsten an 2 und 3 Regionen ( 7 und $6 \mathrm{mal}$ ), am wenigsten an 4,5 und 6 Regionen $(3,3,2 \mathrm{mal})$ und 1,7 und 8 Körperteile waren gar nicht vertreten, und überhaupt fehlten sie ganz in $\mathrm{Nr}$. 22, weil sie hier wahrscheinlich zwischen die erste und zweite Untersuchung fielen oder übersehen worden waren. Zuletzt wurde ein einziger bestehender $W$. in 25 Fällen gesehen: $5 \mathrm{mal} \mathrm{am}$ Arme, $4 \mathrm{mal}$ an der Brust, $2 \mathrm{mal}$ am Beine, $1 \mathrm{mal}$ an der Stirn. 5 mal waren $W$. in je 2 Regionen, 5 mal in je 3 und 2 mal in je 4 vertreten, einmal endlich in allen mit Ausnahme des Nackens, wobei immer wieder die Extremitäten, die Brust und der Rücken vorragen. Wülste waren zuletzt allein unter 26 Fällen $6 \mathrm{mal}$ zu sehen, Z. 3 mal (Nr. 6, 25, 22). In 17 Fällen waren ferner an mehr Körperteilen $Z$. als W. zugegen, 6 mal jedoch mehr W. Bei der letzten Untersuchung überwogen 3 mal die $W$. und 4 mal die $Z$. Man kann wohl sagen, daß im allgemeinen die W. zuletzt auftreten und am längsten dauern. Rechnen wir nun die $Z$. und W. als Gesamtreaktion zusammen, so betrifft sie bei 26 Fällen am häufigsten 8 Gebiete $(7 \mathrm{mal})$, dann 7 G. $(7 \mathrm{mal}), 6 \mathrm{G}$. (5 mal), 3 und $4 \mathrm{Ge}-$ biete je $3 \mathrm{mal}$ und 2 Gebiete $1 \mathrm{mal}$. Also findet eine Gesamtreaktion häufiger eine große als kleine Ausbreitung.

Unter 25 Leichen waren 16 abgemagert (inkl. 1 ödemotöse) und 9 nicht. Unter den ersteren war die Gesamtreaktion $(\mathrm{Z} .+$ W.) in $4 \mathrm{Ge}-$ 
bieten $3 \mathrm{mal}$, in $51 \mathrm{mal}$, in $62 \mathrm{mal}$, in $74 \mathrm{mal}$ und in $86 \mathrm{mal}$, im Durchschnitt in 6,6 Regionen. Bei nicht Abgemagerten in 5 Gebieten 4 mal, in $63 \mathrm{mal}$, in $72 \mathrm{mal}$, im Durchschnitt beinahe in 6 Gebieten. Also war bei Abgemagerten die Gesamtreaktion etwas ausgebreiteter als bei nicht Abgemagerten und wahrscheinlich auch Ödematösen. Freilich ließ sich nicht sicher sagen, ob immer infolge des Leidens Abmagerung stattgefunden hatte. Jedenfalls war kein deutlicher Zusammenhang zwischen ihr, der Krankheitsform und der Todesursache zu ersehen. An den vier mehr oder minder hydropischen Leichen ließ sich keine besondere Beziehung zwischen Ödem und Ausbreitung der Reaktionen erkennen, obgleich letztere a priori geringer sein müßte, wie auch die Stärke der Z. und W.

Man mußte endlich auch fragen, ob der Kadaver in der kalten Jahreszeit seine Muskelvitalität schneller einbüßt, als in der warmen. Unter den 30 Kranken waren in der kalten Jahreszeit (d. h. vom 1. Oktober bis 1. April) 9 gestorben, in der warmen (d. h. vom 1. April bis 1. Oktober) dagegen 21. Bei 26 (Nr. 1, 2, 3, 5 ausgeschaltet) zeigte sich dieselbe durchschnittlich in der kalten Jahreszeit auf 6,6 Regionen ausgebreitet, in der wärmeren aber auf noch nicht ganz 6, so daß kein erheblicher Unterschied bestand, eher sogar paradoxerweise zuungunsten der letzteren. Betrachtete man dagegen die 25 Fälle (Nr. 22 noch ausgelassen) nach der Zeit, wann zuletzt Muskelwülste beobachtet wurden, so zeigte sich ein entschiedenes Ułbergewicht zugunsten der warmen Jahreszeit.

Es waren nämlich in der kalten Jahreszeit bis zu $1 \frac{1}{2}$ Stunden p. m. Wülste zuletzt gesehen bei 6 , in der warmen bei 10 ; in der Zeit bis 2 Stunden 1 resp. 2, in der Zeit bis $2^{1} / 2$ Stunden p. m. 0 resp. 4, darüber 2 resp. 2, wie man es a priori erwartete. Mit der Temperatur geht einigermaßen auch die Totenstarre parallel, aber durchaus nicht streng, noch weniger der Eintritt und die Ausbreitung der Totenflecke. Über beide besitzen wir keine Notizen. Was endlich den ev. Unterschied bez. der Krankheitsform anbelangt, so läßt sich bei unserem Materiale darüber nichts aussagen, da 18 Paralytikern andere Irrseinsformen gegenüberstehen, die meist nur durch einen einzigen Fall vertreten sind.

Was nun die forensische Seite unserer Frage anbetrifft, so werden wir wohl sagen können, da $B$ bei chronischen Geisteskranken, wahrscheinlich auch bei akuten, spätestens nach 4 Stunden, gewöhnlich aber wohl schon nach 3 Stunden p. m. - und zwar scheinbar wenig abhängig von der Außentemperatur, mehr dagegen von der Totenstarre-die mechanischeMuskelerregbarkeit erloschen ist. Bei Normalendürfte es sichähnlich ver halten, was aber noch näher zu untersuchen ist. Diese Bestimmung der verflossenen Zeit durch Feststellung des Aufhörens 
P. Näcke: Die Dauer der postmortalen mechanischen

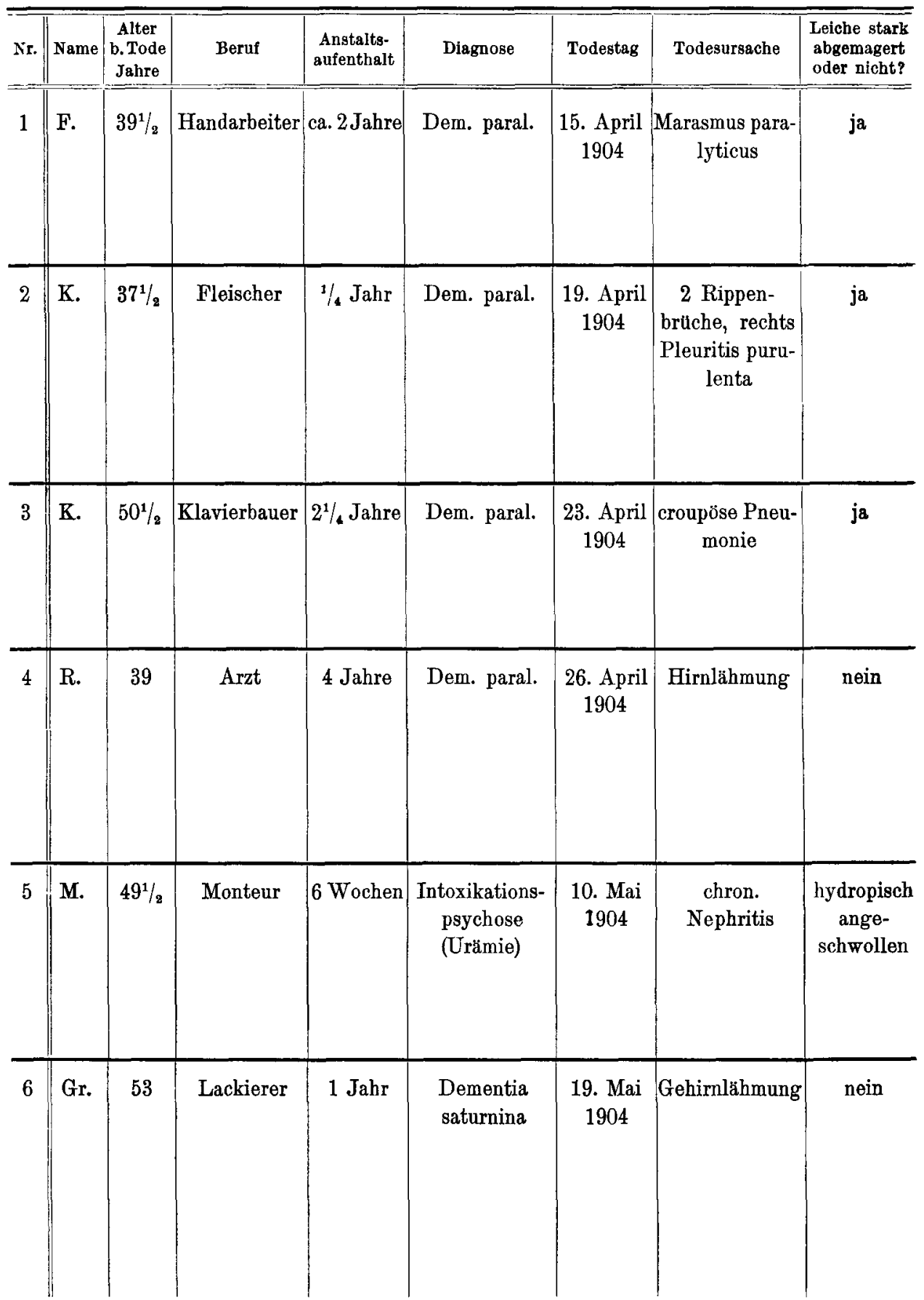

Erklärung der Zeichen: + heißt: Zuckung (Z.) vorhanden; - heißt: sie fehlt; r. = rechts; l. = links; 


\begin{tabular}{|c|c|c|c|c|c|c|c|c|c|}
\hline Untersuchnng & Stirn & $\begin{array}{c}\text { Ge- } \\
\text { sicht }\end{array}$ & $\begin{array}{c}\text { Brust } \\
\text { (Hals) }\end{array}$ & Arme & Beine & 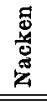 & 氙 & Gesäß & Bemerkungen \\
\hline $\begin{array}{l}1 \% \text { St. p. m. } \\
3^{1} / \text { St. p. m. }\end{array}$ & + & + & $\begin{array}{l}+2 \\
(0) \\
-\end{array}$ & 一 & 一 & $\mathrm{n}$ & $\mathbf{n}$ & $\mathrm{n}$ & $\begin{array}{l}\text { Die Kontraktionen erscheinen nur } \\
\text { als kleine Depressionen an Stirn } \\
\text { und Gesicht. - Links im 5. u. 6. } \\
\text { Intercostalraum unter der Warze } \\
\text { deutlich Kontraktionshtigel. - Ruck- } \\
\text { seite nicht untersucht. Nichts am } \\
\text { Halse zu sehen. Hand und Fub } \\
\text { nicht untersucht. }\end{array}$ \\
\hline $\begin{array}{l}1 / \text { St. p. m. } \\
11 / . \text { St. p. m. } \\
2^{3 / 4} \text { St. p. m. }\end{array}$ & - & $\begin{array}{c}+2 \\
\circ 21 . \\
-\end{array}$ & $\left|\begin{array}{c}+2 \\
021 \\
- \\
-\end{array}\right|$ & $\begin{array}{l}+2 \\
01 \\
- \\
0 \\
-\end{array}$ & $\begin{array}{l}+2 \\
0 \\
- \\
0 \\
-\end{array}$ & $n$ & n & $\mathrm{n}$ & $\begin{array}{l}\text { Deutl. Z. unter d. Jochbogen links, } \\
\text { nicht r. (es war aber dunkel). Nicht } \\
\text { an Hand u. Fuß. . Links außen an der } \\
\text { Wange o. Am Sup. long. I. beim An- } \\
\text { schlagen kontrahieren sich dieFinger, } \\
\text { deutliche Querwülste auf Delt. - Bei } \\
\text { d. 2. Unters. nur schwache o. - Bei } \\
\text { d. 3. Unters. zeigten sich oben am l. } \\
\text { Delt. einige Querwillste, die von der } \\
\text { vorigen Untersuchung wahrscheinl. } \\
\text { stehengeblieben waren. }\end{array}$ \\
\hline $\begin{array}{l}1^{3} \% \text { St. p. m. } \\
2^{3} / \text { St. p. m. }\end{array}$ & 一 & 一 & - & $\begin{array}{l}+ \\
+ \\
0\end{array}$ & + & $\mathrm{n}$ & $\mathbf{n}$ & $\mathrm{n}$ & $\begin{array}{l}\text { Sehr langsame } Z \text {. am } 1 \text {. Delt., nicht } \\
\text { an Oberarmen, wohl aber an Vorder- } \\
\text { armen (Rücken). Innen an beiden } \\
\text { Oberschenkeln schwache Z., nicht } \\
\text { außen, nicht am Untersch. - Bei } \\
\text { der 2. Unters. Z. nur am inneren } \\
\text { Rande des Radius rechts und einige } \\
\text { kleine o auf Delt. }\end{array}$ \\
\hline $\begin{array}{l}10 \text { Min. p. m. } \\
1 \% \text { St. p. m. }\end{array}$ & 一 & - & - & $\begin{array}{l}+2 \\
\circ 1\end{array}$ & + & - & $\begin{array}{l}+ \\
+\end{array}$ & - & $\begin{array}{l}\text { Beim Perkutieren d. Vorderarme } \\
\text { beugt. sich d. Finger. Nichts a. Hand- } \\
\text { rücken od. Bauche. Z. am ganzen } \\
\text { Beine, auch FuBrücken. Am ganzen } \\
\text { Rücken Z., auBer am GesäB, an Ober- } \\
\text { und Unterschenkeln. - Bei der } 2 . \\
\text { Unters. nur viele o auf Vorderf. d. } \\
\text { l. Unterarms, u. beim Pochen am r. } \\
\text { Facialis zuckt es in der Tabatière. } \\
\text { Am Rücken nur leichte Z. in der } 1 . \\
\text { Supraspinalgrube. }\end{array}$ \\
\hline $\begin{array}{l}10 \text { Min. p. } \mathrm{m} \text {. } \\
40 \text { Min. p. m. } \\
\text { fast } 3 \text { St. p. m. }\end{array}$ & $\begin{array}{l}+1 . \\
+1\end{array}$ & 一 & - & + & 一 & $\mathrm{n}$ & $\mathrm{n}$ & $\mathbf{n}$ & $\begin{array}{l}\text { Nur 1. Z. am Corrug. supercil, nicht } \\
\text { am Gesichte. Am Arm nur am Delt. } \\
\text { Beim Beklopfen d. Arme (vorn und } \\
\text { hinten) Z. d. Hände, d. Daumens u. } \\
\text { Abduction. - Bei d. 2. Unters. noch } \\
\text { l. Z, im Corrug., nun auch rechts; } \\
\text { deutlicher Handreflex. - Bei der B. } \\
\text { Unters. Querwuilste am Delt. Leichte } \\
\text { Z. d. Hand u. d. Daumens, sonst } \\
\text { nichts am Arm. }\end{array}$ \\
\hline $\begin{array}{l}12 \text { Min. p. in. } \\
1 \text { St. p. m. }\end{array}$ & - & 一 & +2 & $\begin{array}{l}02 \\
+ \\
02 \\
+\mathbf{r}\end{array}$ & $\begin{array}{l}+ \\
+\mathbf{r}\end{array}$ & - & $\begin{array}{l}- \\
+\end{array}$ & $\begin{array}{l}+2 \\
-\end{array}$ & $\begin{array}{l}\text { Z. am ganzen Orbicul,, sonst nicht. Auf } \\
\text { Front. runde Dellen. Am Halse nicht. } \\
\text { Z.d. Arme bis zum Hand- u. Daumen- } \\
\text { ballen.Z. nur innen am Oberschenkel } \\
\text { u. Wade. Nacken frei, aber Schulter- } \\
\text { blätter nach hinten u. oben gezogen. } \\
\text { Z.derRippen; hinten amoberschenkel } \\
\text { wenig. }-2 \text {, Unters. Z. spurw. im } \\
\text { unteren Augenlid, l. Hand noch etw. } \\
\text { zuckend. Z. auf Waden u. im Inter- } \\
\text { scapularraum. - 3. Unters.: am r. } \\
\text { Vorderarm innen eine leichte, langs. } \\
\text { Z., u. an rechter Wade. }\end{array}$ \\
\hline
\end{tabular}

$\mathbf{Z}_{0}=$ Zuckung; $0=$ Muskelwulst oder Kontraktionshïgel; $\mathrm{n}_{.}=$nicht untersucht; 2, 3 gibt die Stärkegrade an. 
P. Näcke: Die Dauer der postmortalen mechanischen

\begin{tabular}{|c|c|c|c|c|c|c|c|c|}
\hline Nr. & Name & $\begin{array}{c}\text { Alter } \\
\text { b. Tode } \\
\text { Jahre } \\
\end{array}$ & Beruf & $\begin{array}{c}\text { Anstalts- } \\
\text { aufenthalt }\end{array}$ & Diagnose & Todestag & Todesursache & $\begin{array}{l}\text { Leiche stark } \\
\text { abgemagert } \\
\text { oder nicht? }\end{array}$ \\
\hline 7 & A. & $43^{1 / 4}$ & Arbeiter & $1 / 2 \mathrm{Jahr}$ & Dem. paral. & $\begin{array}{c}\text { 19. Juni } \\
1904\end{array}$ & Gehirnlähmung & nein \\
\hline 8 & $\mathrm{~K}$. & $631 \%$ & $\begin{array}{l}\text { Metall- } \\
\text { drücker }\end{array}$ & $1 \mathrm{Jahr}$ & $\begin{array}{c}\text { Melanchol. } \\
\text { senilis }\end{array}$ & $\begin{array}{c}\text { 28. Juni } \\
1904\end{array}$ & $\begin{array}{c}\text { Lungenphthise } \\
\text { Magenkrebs }\end{array}$ & ja \\
\hline 9 & Z. & 60 & ohne & 32 Jahre & $\begin{array}{l}\text { Idiotie mit } \\
\text { Blindheit }\end{array}$ & $\begin{array}{l}\text { 6. Sept. } \\
1904\end{array}$ & $\begin{array}{c}\text { Nephritis } \\
\text { chron. }\end{array}$ & ödematös \\
\hline 10 & H. & $50^{1} / 2$ & Schuhmacher & 3 Wochen & Paralyse & $\begin{array}{l}\text { 7. Sept. } \\
1904\end{array}$ & $\begin{array}{c}\text { Marasmus para- } \\
\text { lyticus }\end{array}$ & ja \\
\hline 11 & It. & $70^{1} / 2$ & Kaufmann & $21 / 4$ Mon. & Dem. senilis & $\begin{array}{l}\text { 11. Sept. } \\
1904\end{array}$ & $\begin{array}{l}\text { Apoplexia } \\
\text { cerebri, } \\
\text { Pneumonie }\end{array}$ & nein \\
\hline 12 & $\mathrm{Br}$. & 39 & Tapezierer & 13 Jahre & $\begin{array}{c}\text { Sekundäre } \\
\text { Demenz } \\
\text { (Dem. praec.) }\end{array}$ & $\begin{array}{c}\text { 17. Sept. } \\
1904\end{array}$ & Phthisis pulm. & ja \\
\hline
\end{tabular}




\begin{tabular}{|c|c|c|c|c|c|c|c|c|c|}
\hline Untersuchung & Stirn & $\begin{array}{c}\text { Ge- } \\
\text { sicht }\end{array}$ & $\begin{array}{c}\text { Brust } \\
\text { (Hals) }\end{array}$ & Arme & Beine & 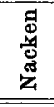 & 承 & Gesä B & Bemerkungen \\
\hline $\begin{array}{l}\text { 1 St. } 5 \text { Min. p. m. } \\
1^{3} / . \text { St. p. m. } \\
2 \% \text { St. p. m. }\end{array}$ & - & - & $\begin{array}{c}+1.0 \\
0 \mathrm{r}\end{array}$ & $\begin{array}{c}0+2 \\
0\end{array}$ & to21. & 一 & $\begin{array}{l}+0 \\
-\end{array}$ & $\begin{array}{c}+2 \\
-\end{array}$ & $\begin{array}{l}\text { Z. spurweise an Brust l.- o auch auf } \\
\text { Sternum. - Nur auf Vorderarm sehr } \\
\text { deutl. Z., nicht Hand. } \\
\text { An Stirn kl. Dellen. - Am Sterno- } \\
\text { cleido-m. vorn r. o. - Am Delt. } \\
\text { waren noch Eindrücke von früher } \\
\text { stehengeblieben. - Am Sternum } \\
\text { noch oben etwas o. } \\
\text { An Stirn noch Dellen, ebenso Ster- } \\
\text { num u. Pector. Deutl. Iange Z. am } \\
\text { l. Quadriceps u. l. Wade. }\end{array}$ \\
\hline $\begin{array}{l}11 / \mathrm{g} \text { St. p. m. } \\
2^{1 / 2} \text { St. p. m. }\end{array}$ & 一 & 一 & $\begin{array}{l}+0 \\
+0\end{array}$ & $+2 \circ$ & $\begin{array}{l}+o \\
+\end{array}$ & - & 一 & - & $\begin{array}{l}\text { Ganz geringe Z. am Corrug. supercil. } \\
\text { u. etwas unter d. Auge. An An } \\
\text { Brust bes. I. Z. - Zwisch. d. Rippen } \\
\text { Wuilste. Deutl. Z. bis in Hand-: } \\
\text { Kniescheibe schneilt empor u. l. d. } \\
\text { FuB. Sehr deutl. Z. zwischen und } \\
\text { unterhalb der Scapula. } \\
\text { Auf Sternum kl. O. - Bis in d. Hand } \\
\text { leichte Z., auBer r. Antithenar. - } \\
\text { Ganz leichte Z. noch zwischen den } \\
\text { Schulterblättern. } \\
\text { Noch leichte Z. im l. Thenar. - An } \\
\text { Beinen nur an Wade leichte Z. }\end{array}$ \\
\hline 2 St. p. m. & o & - & o & - & - & - & - & - & $\begin{array}{l}\text { Nirgends Z. Nur über Stirn u. Clavi- } \\
\text { cula leichte Dellenbildung. }\end{array}$ \\
\hline $\begin{array}{l}1 / 2 \text { St. p. m. } \\
11 / 2 \text { St. p. m. }\end{array}$ & - & 一 & $\begin{array}{c}+ \\
0+r\end{array}$ & $\begin{array}{c}+ \\
02+\end{array}$ & - & 一 & - & $\begin{array}{l}+ \\
-\end{array}$ & $\begin{array}{l}\text { Oberflächl. u. tiefe Nackenmuskeln } \\
\text { zeigen Z., u. Scap. hebt sich. - Z. } \\
\text { bis in Hand. } \\
\text { Nur rechts b. Perkussion d. Pect. } \\
\text { Heben d. Axillarfalte; am Delt. } \\
\text { breite, } 3 \mathrm{~cm} \text { lange quere Wülste } \\
\text { lange bleibend. } \\
\text { Auf Rücken und Schulterblätter } \\
\text { kleine Eindrücke - vielleicht in } \\
\text { Nacken u. Wade Spuren von } Z \text {. }\end{array}$ \\
\hline $\begin{array}{l}11 / \mathrm{g} \text { St. p. m. } \\
21 / 4 \text { St. p. m. }\end{array}$ & 0 & - & $\begin{array}{c}+0 \\
0\end{array}$ & $\begin{array}{c}20- \\
0\end{array}$ & $\begin{array}{c}+ \\
0+\end{array}$ & - & $\begin{array}{l}+o \\
- \\
-\end{array}$ & 一 & $\begin{array}{l}\text { Kleine Dellen auf Corrig. }-\mathrm{Z} \text { im } \\
\text { Pect. rechts, l. fast nicht. }- \text { An } \\
\text { beiden Armen mehr Z. als im Delt. } \\
\text { - Am Thenar Dellen. An Schulter- } \\
\text { blättern keine Z., aber oberhalb u. } \\
\text { an Rippen. } \\
\text { An Armen fast nur lange } \circ, 1 .>\mathrm{r} \text {. } \\
\text { Am Kinn noch kl. } 0 \text {. - Am Bauch } \\
\text { noch deutl. Dellen. - Rechts seit- } \\
\text { lich unten von Patella einige kurze } Z \text {. }\end{array}$ \\
\hline $\begin{array}{l}1^{1 / 2} \text { St. p. m. } \\
2^{1 / 4} \text { St. p. m. } \\
\text { 3 St. p. m. }\end{array}$ & $\begin{array}{l}+ \\
- \\
-\end{array}$ & $\begin{array}{l}+ \\
- \\
-\end{array}$ & $\begin{array}{c}+0 \\
0 \\
-\end{array}$ & $\begin{array}{c}+0 \\
0 \\
0\end{array}$ & $\begin{array}{l}+0 \\
+1 \\
+021\end{array}$ & $\begin{array}{l}- \\
-\end{array}$ & $\begin{array}{l}- \\
+ \\
-\end{array}$ & $\begin{array}{l}+ \\
+ \\
+\end{array}$ & $\begin{array}{l}\text { Kl. Gesichtsmuskeln Z., auch Kinn } \\
\text { u. am unteren Teile d. Orbicul. oculi. } \\
\text { Z. fast nur an Vorderarmen und } \\
\text { Thenar. - Leichte Z. im Suprascapul. } \\
\text { nur. - Am ganz. Glutaeus sehr deut- } \\
\text { liche Eindrücke. } \\
\text { Spur von Z. am Orbicul. oculf inf. } \\
\text { und Gesicht. } \\
\text { Noch am Iliopsoas l. leichte Z. - R. } \\
\text { am Bein keine Z. mehr. } \\
\text { Am Oberschenkel innen schwache Z., } \\
\text { 1. breite O. }\end{array}$ \\
\hline
\end{tabular}




\begin{tabular}{|c|c|c|c|c|c|c|c|c|}
\hline Nr. & Name & \begin{tabular}{|c|} 
Alter \\
b.Tode \\
Jahre \\
\end{tabular} & Beruf & $\begin{array}{c}\text { Anstalts- } \\
\text { aufenthalt }\end{array}$ & Diagnos $\theta$ & Todestag & Todesursache & $\begin{array}{l}\text { Leiche stark } \\
\text { abgemagert } \\
\text { oder nicht? }\end{array}$ \\
\hline 13 & E. & 71 & Handarbeiter & 19 Jahre & $\begin{array}{c}\text { Sekundäre } \\
\text { Demenz } \\
\text { (Dem. praec.) }\end{array}$ & $\begin{array}{l}\text { 19. Nov. } \\
1904\end{array}$ & $\begin{array}{c}\text { Magenkrebs } \\
\text { Phthise }\end{array}$ & ja \\
\hline 14 & $\mathrm{Gr}$. & 47 & Schriftsetzer & ca. $11 \mathrm{~J}$. & $\begin{array}{c}\text { Halluzina- } \\
\text { torische } \\
\text { Verrücktheit } \\
\text { (Dem. praec.) }\end{array}$ & $\begin{array}{c}\text { 28. Dez. } \\
1904\end{array}$ & Vitium cordis & $\begin{array}{c}\text { nein } \\
\text { ödematös }\end{array}$ \\
\hline 16 & St. & 47 & Drogist & $4^{1} / 2$ Jahre & Dem. paral. & $\begin{array}{l}\text { 30. Aug. } \\
1905\end{array}$ & $\begin{array}{l}\text { Erstickung } \\
\text { durch Ver- } \\
\text { schlucken }\end{array}$ & ja \\
\hline 16 & M. & $58^{1 / 2}$ & Barbier & $1 \mathrm{Jahr}$ & Dem. paral. & $\begin{array}{l}\text { 1. April } \\
1905\end{array}$ & Hirnlähmung & ja \\
\hline 17 & $\mathrm{~K}$. & 48 & $\begin{array}{l}\text { Tabak- } \\
\text { arbeiter }\end{array}$ & 6 Jahre & Dem. paral. & $\begin{array}{l}\text { 2. April } \\
1905\end{array}$ & Pneumonie & nein \\
\hline 18 & N. & $33^{3 / 4}$ & Handarbeiter & $21 / 4$ Jahre & $\left\{\begin{array}{c}\text { Pseudoparalyse } \\
\text { (Blei) }\end{array}\right.$ & $\begin{array}{l}\text { 4. April } \\
1905\end{array}$ & $\begin{array}{l}\text { Phthisis } \\
\text { pulmon. et } \\
\text { intestin. }\end{array}$ & ja \\
\hline 19 & $G$. & 47 & Maurer & $2^{1} / 2$ Jahre & Paralyse & $\begin{array}{c}\text { 12. Mai } \\
190 . j\end{array}$ & $\begin{array}{l}\text { Pneu- } \\
\text { monie }\end{array}$ & ja \\
\hline 20 & F. & 45 & Tischler & 15 Jahre & Dem. praec. & $\begin{array}{l}\text { 9. Juli } \\
1905\end{array}$ & $\begin{array}{l}\text { Phthisis } \\
\text { pulmon. }\end{array}$ & ja \\
\hline
\end{tabular}




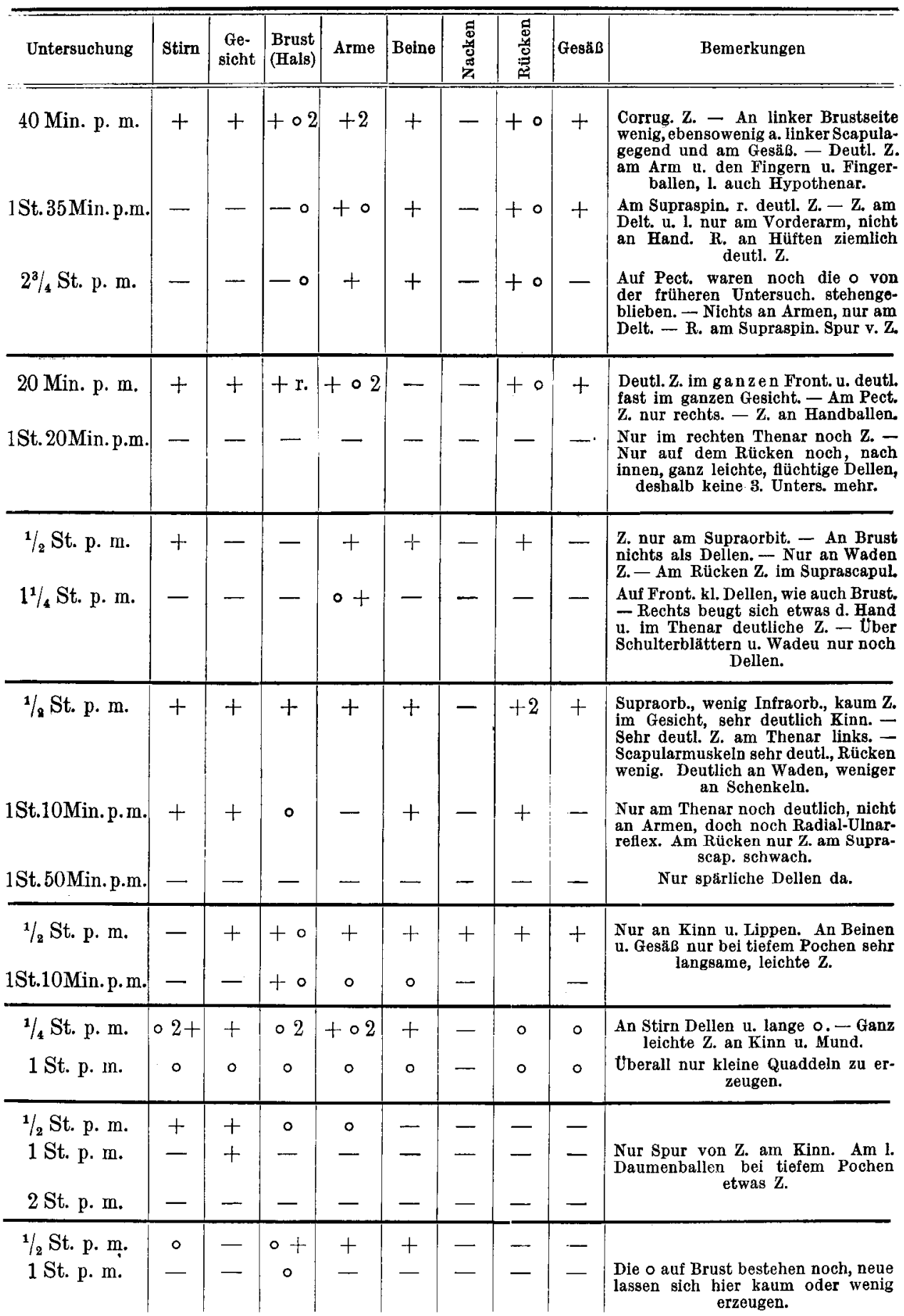


P. Näcke: Die Dauer der postmortalen mechanischen

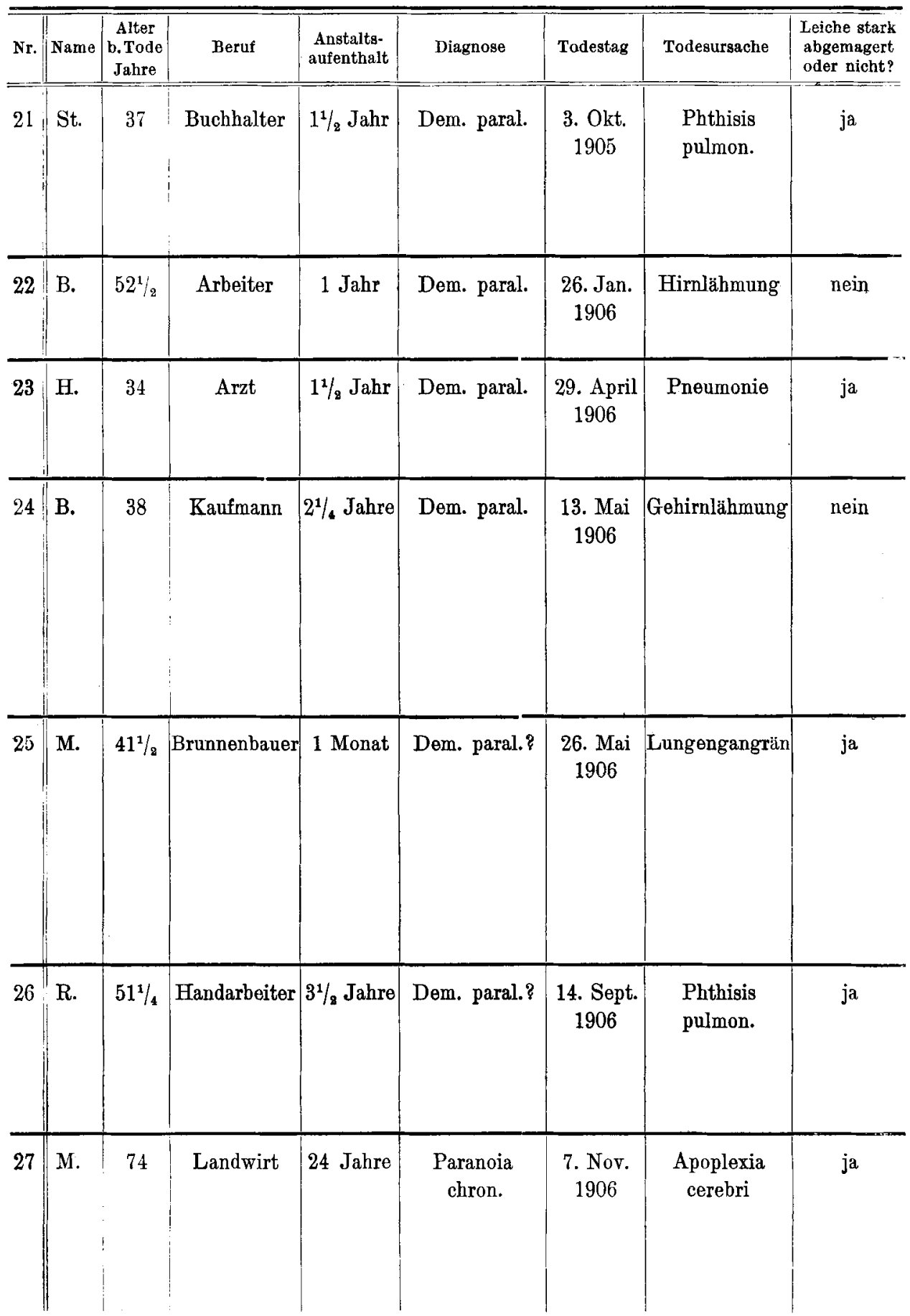




\begin{tabular}{|c|c|c|c|c|c|c|c|c|c|}
\hline Untersuchung & Stirn & $\begin{array}{l}\text { Ge- } \\
\text { sicht }\end{array}$ & $\begin{array}{l}\text { Brust } \\
\text { (Hals) }\end{array}$ & Arme & Beine & 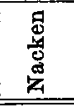 & 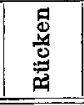 & Gesäß & Bemerkungen \\
\hline 25 Min. p. m. & + & + & +0 & + & +2 & + & +0 & +2 & Am Kinn tiberall $\mathbf{Z}$ \\
\hline 1 St. 7 Min. p. m. & + & + & + & $\circ 2+$ & + & + & + & + & $\begin{array}{l}\text { Noch ziemlich deutl. Z. am Supra- } \\
\text { spin. und zwischen Schulterblättern, } \\
\text { nicht mehr am Infraspin. }\end{array}$ \\
\hline 2 St. p. m. & - & - & 一 & 一 & $\circ$ & - & + & 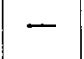 & $\begin{array}{l}\text { Nur noch am Lev. scap. und am } \\
\text { Thenar. }\end{array}$ \\
\hline 1 St. p. m. & - & + & + & + & + & - & + & 一 & $\underset{\text { Nur am Kinn. }}{\text { frascapul. }}$ Spur v. Z. am In- \\
\hline 2 St. p. m. & 一 & - & - & - & 一 & - & - & - & Vielfache Dellen an den Gliederm. \\
\hline $1 /$ St. p. m. & - & +0 & 0 & ० & + & - & 0 & o & $\begin{array}{l}\text { Nur Z. am unteren Orbicul. oculi } \\
\text { u. Kinn. An Armen keine eigenti. } \\
\text { Z., sondern es kontrahieren sich d. } \\
\text { Fingerbeuger. }\end{array}$ \\
\hline $11 /$ St. p. m. & 一 & + & $\circ$ & $\circ$ & $\circ$ & 一 & - & - & \\
\hline $1 / \mathrm{st}$. p. m. & + & + & + & +2 & + & 一 & +2 & +2 & $\begin{array}{l}\text { An Waden stärkere, an Oberschenkel } \\
\text { kaum Z. da. Nicht an Brust, aber } \\
\text { am Platysma (?). }\end{array}$ \\
\hline 1 St. p. m. & + & + & + & + & + & 一 & + & + & $\begin{array}{l}\text { Am Rücken Z. nur zwischen } \\
\text { Schulterblättern. }\end{array}$ \\
\hline $1^{3} /$ St. p. m. & - & + & + & +02 & + & 一 & + & - & $\begin{array}{l}\text { Spuren am Kinn, am Platysma (?). } \\
\text { Zwischen den Schulterblattern Z., u. } \\
\text { rechte Schulter hob sich noch. Im } \\
\text { 1. Intercostalraum etwas } Z \text {. }\end{array}$ \\
\hline 2 St. p. In. & 一 & + & + & +0 & + & 一 & + & - & Alles in Spuren, nur in 1. Wade \\
\hline $1 /$ St. p. m. & + & + & $+\circ 2$ & + & + & +20 & +20 & +20 & $\begin{array}{l}\text { Spurw. an langen Bauchmuskeln } Z \text {. } \\
\text { Deutl. auch an Fußrücken (Zehen } \\
\text { heben sich). Sehr deutlich am } \\
\text { Sternocleid., Schulterblättern und } \\
\text { Seiten. }\end{array}$ \\
\hline$\%$ St. p. m. & +0 & - & 0 & 0 & + & + & +0 & + & $\begin{array}{l}\text { Nur am Thenar Z. - Deutlich am } \\
\text { Sternocleid. u. zwischen d. Schulter- } \\
\text { blättern. }\end{array}$ \\
\hline $1^{3} / 4$ St. p. m. & - & - & 一 & - & + & + & $t$ & + & $\begin{array}{l}\text { Auf Stirn, an Arm u. Rlicken waren } \\
\text { die o stehen geblieben von der } \\
\text { früheren Untersuchung her, aber } \\
\text { keine neue zu erzeugen. }\end{array}$ \\
\hline 20 Min. p. m. & + & + & + & $+o$ & +2 & + & +0 & + & $\begin{array}{l}\text { Spur an d. Stirn, auch an l. Inter- } \\
\text { costalmuskeln. Am Rücken nur } \\
\text { zwischen Schuiterblättern, kaum an } \\
\text { ihnen selbst. }\end{array}$ \\
\hline 55 Min. p. m. & + & + & - & 0 & +0 & - & +0 & - & Am Arm Z. nur an Daumenballen. \\
\hline St. 28Min.p.m. & - & - & 一 & $\circ$ & o & - & & - & Nur noch kl. $\circ$ zu erzeugen. \\
\hline 25 Min. p. m. & +2 & + & $+o 3$ & $+2 \circ 2$ & + & + & + & + & $\begin{array}{c}\text { Augenbrauen stark emporgehoben } \\
\text { durch Schlag. - Am Sternocleid. Z. } \\
\text { vorn u. hinten. - Am Biceps starke } \\
\text { Z. u. } 0 \text {. }\end{array}$ \\
\hline 1 St. p. m. & - & $t$ & + & $+\circ 2$ & - & - & 一 & + & $\begin{array}{l}\text { Nur arn Kinn Spur. }-\underset{\text { Am Biceps }}{\text { noch ziemlich deutlich. }}\end{array}$ \\
\hline $11 / 2$ St. p. m. & - & + & o & +0 & - & 一 & -0 & - & $\begin{array}{l}\text { Seitlich am Kinn noch Spuren von } \\
\text { Z. - t'ber d. Suprascap. vielleicht } \\
\text { einige ganz' leichte } \mathrm{Z} \text {. }\end{array}$ \\
\hline
\end{tabular}




\begin{tabular}{|c|c|c|c|c|c|c|c|c|}
\hline Nr. & Name & \begin{tabular}{|c|} 
Alter \\
b. Tode \\
Jahre
\end{tabular} & Beruf & $\begin{array}{c}\text { Anstalts- } \\
\text { aufenthalt }\end{array}$ & Diagnose & Todestag & Todesursache & $\begin{array}{l}\text { Leiche stark } \\
\text { abgemagert } \\
\text { oder nicht? }\end{array}$ \\
\hline 28 & $\mathrm{H}$. & 49 & Maurer & $\begin{array}{l}12 \text { Jahre } \\
\text { (mit Unter- } \\
\text { brechung) }\end{array}$ & $\begin{array}{l}\text { chron. halluzin. } \\
\text { Verrucktheit }\end{array}$ & $\begin{array}{c}\text { 16. Febr. } \\
1907\end{array}$ & $\begin{array}{l}\text { Phthisis } \\
\text { pulmon. }\end{array}$ & ja \\
\hline 29 & G. & $41^{3 / 4}$ & $\begin{array}{l}\text { Handschuh- } \\
\text { zuschneider }\end{array}$ & $3^{1} / 4$ Jahre & Dem. paral. & $\begin{array}{c}\text { 2. Sept. } \\
1907\end{array}$ & Paralyt. Anfall & $\begin{array}{c}\text { ja } \\
\text { Ödem }\end{array}$ \\
\hline 30 & P. & 45 & Arbeiter & $4^{1 / 2}$ Jahre & Dem. paral. & $\begin{array}{l}\text { 27. Juli } \\
1908\end{array}$ & Hirnlähmung & nein \\
\hline
\end{tabular}

des Muskellebens ist vielleicht in concreto einmal nützlich und jedenfalls sicherer als die nach der Zeit des Auftretens von Totenstarre und Totenflecken, ihrer Stärke und Ausbreitung, die jedenfalls größeren Variationen ausgesetzt sind.

Unter nochmaliger Hervorhebung des Umstandes, daß bei unserer Untersuchung so manche Fehlerquellen vorlagen und vor allem jeder Vergleich mit Normalen aussteht, sind trotzdem gewisse Richtungslinien nicht $\mathrm{zu}$ verkennen, welche recht wohl zur Orientierung für Nachuntersuchungen dienen können. Unsere Hauptergebnisse selbst lassen sich aber folgendermaßen zusammenfassen:

1. Die zuletzt beobachteten Muskelzuckungen infolge von Perkussion waren am frühesten $1 / 2$ bis 1 Stunde post mortem, am spätesten $23 / 4$ Stunden, am häufigsten zwischen 2-3 Stunden post mortem. Die Muskelwülste traten gewöhnlich später auf und endigten meist später. Die gesamte Múskelerregbarkeit (Zuckungen und Wülste usw.) dürfte in 3-4 Stunden gänzlich verschwinden.

2. Die meisten Zuckungen betrafen die Extremitäten und die Brust; die wenigsten Stirn und Nacken. Nicht immer waren sie symmetrisch.

3. Dem entspricht im allgemeinen auch die Stärke der Zuckung, mit Ausnahme des Gesäßes.

4. Der gleichen Regel folgte meist auch die Ausbreitung derselben an derselben Körperpartie, und die Stärke schien damit Hand in Hand zu gehen. 
Muskelerregbarkeit bei chronischen Geisteskranken, speziell Paralytikern.

\begin{tabular}{|c|c|c|c|c|c|c|c|c|c|}
\hline Untersuchung & Stirn & $\begin{array}{c}\text { Ge- } \\
\text { sicht }\end{array}$ & $\begin{array}{l}\text { Brust } \\
\text { (Hals) }\end{array}$ & Arme & Beine & 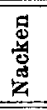 & 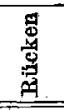 & Gesä B & Bemerkungen \\
\hline $1 / 2$ St. p. m. & 0 & + & 。 & +2 & $\circ$ & - & + & $\circ 2$ & $\begin{array}{l}\text { Starke Z. am rechten Biceps und } \\
\text { Vorderarm, wenig links. - Deutliche } \\
\text { Z. zwischen Schulterblättern, auf } \\
\text { ihnen wenig. }\end{array}$ \\
\hline 1 Std. p. m. & $\circ$ & 一 & o & -0 & 一 & - & - & 一 & \\
\hline 25 Min. p. m. & + & +2 & + & +2 & + & + & +2 & +2 & $\begin{array}{l}\text { An der Stirn vielfach fibrilläre Z. u. } \\
\text { Heraufziehen d. Augenbrauen.-Am } \\
\text { Kinn sehr starke Z. - Längs des des } \\
\text { ganzen Rückens Z. deutlieh. An d. } \\
\text { odematösen Schenkeln weniger als } \\
\text { an d. Unterschenkeln. }\end{array}$ \\
\hline 55 Min. p. m. & $0+$ & + & +0 & + & + & + & + & + & $\begin{array}{l}\text { Noch am Rücken deutl. Z, nicht am } \\
\text { Bauch (auch vorher nicht). }\end{array}$ \\
\hline 1St.40Min.p.m. & ○ & + & + & + & + & - & + & - & \\
\hline 20 Min. p. m. & 。 & + & + & + & + & + & + & 一 & An Armen am deutlichsten. \\
\hline 55 Min. p. m. & $\circ$ & - & - & + & + & + & + & - & $\begin{array}{c}\text { Spuren noch am Delt., vielleicl t } \\
\text { auch Biceps. }\end{array}$ \\
\hline 1St. 25Min.p.m. & $\circ$ & - & ० & 一 & + & + & + & - & $\begin{array}{l}\text { Im Delt. ganz schwache } Z_{\text {, }} \text { nicht } \\
\text { mehr im Biceps. }\end{array}$ \\
\hline
\end{tabular}

5. Die Zuckungen waren am häufigsten über $6-8$ Körpergegenden verbreitet, am meisten über 8 , und damit ging im allgemeinen auch die Stärke der Ausschläge parallel.

6. Die Ausbreitung und Stärke der Muskelwülste ging im allgemeinen der der Zuckungen parallel. Sie waren also am häufigsten an Extremitäten und Brust, während sie sich, im Gegensatze zu den Zuckungen, am häufigsten über 2-3 Regionen verbreiteten. Sie schienen nicht an allen Muskeln vorzukommen.

7. Ein einziger Muskelwulst erschien zuletzt an den Extremitäten und an der Brust, und auch bei gruppenweisem, letztem Auftreten prävalierten diese Teile.

8. Die Gesamtreaktion (Zuckungen und Wülste) ist häufiger weit als nur wenig ausgebreitet.

9. Bei abgemagerten Leichen waren Ausbreitung und Stärke der Gesamtreaktion etwas größer als bei nicht abgemagerten.

10. Dasselbe läßt sich bez. der Außentemperatur beim Tode sagen. In der Wärme hielt die Gesamtreaktion länger aus als in der Kälte. Mit zunehmender Totenstarre nahmen alle Erscheinungen ab.

11. Bezüglich des Unterschiedes bei Paralyse und anderen Psychosen ließ sich nichts aussagen, da von letzteren meist nur je 1 Fall vorlag.

12. Forensisch nicht unwichtig erscheint vielleicht die Folgerung aus unseren Studien, daß 3-4 Stunden p. m. keine mechanische Muskelerregbarkeit mehr nachweisbar war, 
446 P. Näcke: Die Dauer der postmortalen mechanischen Muskelerregbarkeit usw.

und das scheinbar wenig abhängig von der Außentemperatur, wohl aber sehr vom Eintritt und vom Grad der Totenstarre. Jedenfalls erscheint diese Zeitbestimmung sicherer als die viel variablere nach Eintritt und Ausbreitung der Totenstarre oder gar der Totenflecke. Bei akuten Geisteskranken werden sich die Verhältnisse kaum anders gestalten, und a uch von den geistig Gesunden dürfte ein Gleiches zu erwarten sein, wenngleich bei Irren überhaupt, besonders aber bei chronischen, die Muskelvitalität öfter schon mehr oder weniger schwer geschädigt sein dürfte. 